\title{
Simulation of Relativistic Shocks and Associated Self-consistent Radiation
}

\author{
K.-I. Nishikawa ${ }^{1}$, J. Niemiec ${ }^{2}$, M. Medvedev ${ }^{3}$, B. Zhang ${ }^{4}$, P. Hardee ${ }^{5}$, Y. Mizuno ${ }^{1}$, \\ A. Nordlund ${ }^{6}$, J. Frederiksen ${ }^{6}$, H. Sol ${ }^{7}$, M. Pohl ${ }^{8}$, D. H. Hartmann ${ }^{9}$, G.J. Fishman ${ }^{10}$ \\ ${ }^{1}$ Center for Space Plasma and Aeronomic Research, University of Alabama in Huntsville, \\ NSSTC, 320 Sparkman Drive, Huntsville, AL 35805, USA; \\ ${ }^{2}$ Institute of Nuclear Physics PAN, ul. Radzikowskiego 152, 31-342 Krakow, Poland \\ ${ }^{3}$ Department of Physics and Astronomy, University of Kansas, KS 66045, USA \\ ${ }^{4}$ Department of Physics and Astronomy, University of Nevada, Las Vegas, NV 89154, USA \\ ${ }^{5}$ Department of Physics and Astronomy, The University of Alabama, Tuscaloosa, AL 35487, \\ USA \\ ${ }^{6}$ Niels Bohr Institute, Juliane Maries Vej 30, 2100 København Ø, Denmark \\ ${ }^{7}$ LUTH, Observatore de Paris-Meudon, 5 place Jules Jansen, 92195 Meudon Cedex, France \\ ${ }^{8}$ Institut fuer Physik und Astronomie, Universitaet Potsdam, 14476 Potsdam-Golm, Germany \\ ${ }^{9}$ Department of Physics and Astronomy, Clemson University, Clemson, SC 29634, USA \\ ${ }^{10}$ NASA/MSFC, 320 Sparkman Drive, Huntsville, AL 35805, USA
}

Recent PIC simulations of relativistic electron- positron (electron- ion) jets injected into a stationary medium show that particle acceleration occurs at shocked regions. Simulations show that the Weibel instability is responsible for generating and amplifying highly nonuniform, small-scale magnetic fields and particle acceleration. These magnetic fields contribute to the electron's transverse deflection behind the shock. The "jitter" radiation from deflected electrons in turbulent magnetic fields has different properties than synchrotron radiation, which is calculated in a uniform magnetic field. This jitter radiation may be important for understanding the complex time evolution and/or spectral structure in gamma-ray bursts, relativistic jets in general, and supernova remnants. We will present detailed spectra for conditions relevant of various astrophysical sites of shock formation via the Weibel instability. In particular we will discuss the application to GRBs and SNRs. 